\title{
Review of: "Evaluation of the frailty characteristics and clinical outcomes according to the new frailty- based outcome prediction model (Myeloma Risk Profile-MRP) in a UK real-world cohort of elderly newly diagnosed Myeloma patients"
}

\author{
Sascha Tuchman ${ }^{1}$ \\ 1 University of North Carolina at Chapel Hill
}

Potential competing interests: Honoraria / advisory board: Janssen; Research funding: Sanofi

In their publication "Evaluation of the frailty characteristics and clinical outcomes according to the new frailty-based outcome prediction model (Myeloma Risk Profile-MRP) in a UK real-world cohort of elderly newly diagnosed Myeloma patients," Djebbari et al. present data regarding the evaluation of the riskstratification system, the Myeloma Risk Profile (MRP) - a scored system built on age, WHO performance status, C-reactive protein, and international staging system (ISS) - in a cohort of 100 patients with newly diagnosed multiple myeloma (MM) treated with weekly, fixed duration bortezomib, cyclophosphamide and dexamethasone (VCD). Aside from their MRP score, included patients were generally clinically high-risk; median age was in the 70 's, and $>50 \%$ of patients had MM that was associated with ISS 3 , high-risk genetics, elevated LDH, and/or World Health Organization (WHO) performance status of 2 or worse. Data were collected retrospectively. Standard clinical outcomes were assessed, such as progression-free and overall survival (PFS and OS respectively), overall response rates (ORR), and adverse events. Due to small numbers, MRP-intermediate and -fit patients were pooled, and frailty characteristics and disease outcomes were compared between that pooled group and MRP-frail patients.

Outcomes were relatively poor and discontinuation was frequent, which is unsurprising for a real-world study of high-risk patients; real-world patients often are older, and have more co-morbidities, and worse performance status than the more pristine patients that are typically enrolled on prospective clinical studies. Only approximately $50 \%$ of patients completed the planned therapy. ORR across the entire cohort was 75\% (reasonable), but median PFS was $<1$ year. OS has not been reached, largely because median follow-up is still relatively short at less than three years. No significant differences in these outcomes were observed between the frail and intermediate/fit cohorts at data cutoff. Uni- and multivariate analyses were attempted in an effort to identify predictors of both PFS and OS, but none met statistical significance including MRP. Lastly, adverse events were reviewed and observed to be uncommon in general, including even Common Terminology Criteria for Adverse Events (CTCAE) grade 1 
hematological events. Peripheral neuropathy and infections were the exception, with each occurring in roughly one third of patients. It appeared that adverse events were more frequent in MRP-frail patients.

With this publication, Djebbari et al. build on published data describing MRP as a practical and relevant risk stratification tool, and they add an important real-world perspective to existing UK data regarding MRP, which was created and primarily validated using populations from prospective clinical trials. The fact that all patients were treated uniformly and at a relatively small number of centers reduces heterogeneity and mitigates the frequent confounders that arise in other retrospective studies, that stem from differences in chemotherapy selection and institutional supportive care protocols. Although the data derived from this study are mostly exploratory, they provide a useful, preliminary glimpse into the types of information MRP could give when employed for use in routine clinical care outside of prospective studies.

Two important critiques warrant mention:

- Sample size: As the authors discuss themselves, the relatively small sample size of the study population limits what can be learned from this study. It was noted that adverse events appeared to be more common in frail patients. Beyond that, however, the fact that none of even the known, strong prognostic factors in MM, such as ISS stage 3 or high-risk genetics, were significant, even univariate predictors of outcome in this study, demonstrates that the study was seriously underpowered. Given that the study was largely intended to examine MRP's capacity to stratify patients, and categorization of frail vs. intermediate/fit by MRP did not meaningfully predict any outcomes of interest beyond possibly adverse events, further supports this critique. In short, it is difficult to know how to apply these data when the primary instrument of interest demonstrated a limited ability to do what it was designed to do, and as it has successfully done in the other, larger studies that preceded this one. The authors appropriately cite this as a caveat and note that their observations regarding longer-term outcomes are exploratory, not definitive. Their suggestion that this study should provide impetus for larger studies is apt.

- External validity: Although as mentioned the real-world, uniformly treated nature of the studied population increases external validity, the actual treatment regimen that subjects received detracts from it. Fixed-duration VCD for clinically high-risk MM is fairly far from how MM would typically be managed in 2022, even among older, frail adults, given the results of numerous studies such as ALCYONE(1), MAIA(2) and FIRST(3), which have all robustly proven the utility of and provided the basis for regulatory approval in some countries of the combinations of daratumumab, melphalan, bortezomib, and prednisone; lenalidomide and dexamethasone; and daratumumab, lenalidomide and dexamethasone, respectively. All those studies included some component of continuous therapy and all have shown OS benefits as compared to the control arms. The facts that MM therapy has advanced far beyond fixed-duration VCD, and sample size was small, make it challenging to know exactly how to 
frame data presented here in the larger context and envisage how MRP could function nowadays. That is no fault of the authors, rather it is a promising commentary on how quickly MM therapeutics are evolving and improving. Things done just a few years ago drift toward obsolescence soon thereafter.

In summary, Djebbari et al. are to be commended for testing the MRP's ability to risk stratify a real-world population of MM patients, and as such to assist us all with the difficult task of bridging the gap from ivory tower research to practical implementation. The exploratory data they provide give a glimpse into how MRP performs when applied outside of the relatively sterile world of prospective clinical trials. Although the reader must view the data as exactly that - exploratory - this study will hopefully give rise to larger studies examining MRP when applied real-world patients treated on more modern regimens.

\section{References:}

- 1. Mateos MV, Dimopoulos MA, Cavo M, Suzuki K, Jakubowiak A, Knop S, et al. Daratumumab plus Bortezomib, Melphalan, and Prednisone for Untreated Myeloma. N Engl J Med. 2018;378(6):518-28.

- 2. Facon T, Kumar S, Plesner T, Orlowski RZ, Moreau P, Bahlis N, et al. Daratumumab plus Lenalidomide and Dexamethasone for Untreated Myeloma. N Engl J Med. 2019;380(22):2104-15.

- 3. Facon T, Dimopoulos MA, Dispenzieri A, Catalano JV, Belch A, Cavo M, et al. Final analysis of survival outcomes in the phase 3 FIRST trial of up-front treatment for multiple myeloma. Blood. 2018;131(3):301-10. 